\title{
Tip optical fiber refractive index sensor based on a thin copper film
}

\author{
Y. Lopez-Dieguez ${ }^{a}$, J. M. Estudillo-Ayala ${ }^{b}$, D. Jauregui-Vazquez ${ }^{b, *}$, L. A. Herrera-Piad ${ }^{b}$, J. A. Martín-Vela ${ }^{b}$ \\ J. M. Sierra-Hernandez ${ }^{b}$, J. C. Hernandez-Garcia ${ }^{b, c}$, M. Bienchetti ${ }^{b}$, M. Avazpour ${ }^{b}$ and R. Rojas-Laguna ${ }^{b}$ \\ ${ }^{a}$ División de Ingeniería Electrónica, Tecnológico de Estudios Superiores de Ixtapaluca, \\ Ixtapaluca, Km. 7 Carr. Ixtapaluca-Coatepec San Juan, Coatepec Ixtapaluca Mexico, 56580. \\ ${ }^{b}$ División de Ingenierías Campus Irapuato Salamanca, \\ Departamento de Electrónica, Universidad de Guanajuato, Salamanca 36885, México. \\ *e-mail: jaureguid@ugto.mx \\ ${ }^{c}$ Consejo Nacional de Ciencia y Tecnología, \\ Del. Benito Juárez 039040, México.
}

Received 29 November 2020; accepted 13 December 2020

\begin{abstract}
We report a refractive index tip fiber optic sensor based on a modal interferometer coated with a thin copper film. This device can be used for solute concentration in aqueous concentration measurement. The copper film increases the device reflectivity and, as a result, increases the multimodal interference pattern visibility. To validate the sensor device, different water-glycerol weight concentrations were used in the range from 0 to 45 Glycerol \%. The recorded spectral responses show a redshifting, and a sensitivity of $19 \mathrm{pm} /$ (Glycerol \% by weight) from 0 to $18 \%$ and $4.7 \mathrm{pm} /$ (Glycerol \% by weight) from 18 to $45 \%$ was achieved. Besides, using a phase analysis, we calculated a sensitivity of $-15 \mathrm{rad} / \mathrm{RIU}$.
\end{abstract}

Keywords: Fiber optic sensor; modal fiber optic Interferometer; liquid concentration. Measurement.

PACS: 78.66.-w; 78.40.-q

\section{Introduction}

Fiber optics sensors have been an attractive alternative for sensing several physical parameters [1-10]. Moreover, solute concentration in an aqueous solution is a chemical parameter that is very useful in many industrial processes. Most published works were centered on the refractive index measurement [11-15]. Also, researchers were interested in measuring the solute concentration in aqueous solutions [15-19]. Some methods to determine solute concentration are based on the relationship between refractive index and solute concentration $[16,17,19,20]$. As a consequence, several fiberoptic-sensors have been proposed to detect this parameter, for instance: tapered fibers [15], fused biconical fiber couplers [16], single-mode - multimode - single-mode fiber structure cascaded with a fiber Bragg grating [17], Surface plasmon resonance (SPR) [18], and a setup based on two optical fibers with sensing ends using the Fresnel reflection technique [19]. It is important to mention that some procedures are complicated, and the materials involved are expensive [12,15-17].

In previous work, we reported some similar structures for refractive index sensing using intensity modulation detection [13]. In the present report, we improve one of those structures using a thin copper film-coated for solute concentration in aqueous solutions sensing using the phase modulation detection. The intensity modulation detection offers a low-cost demodulation process, but the measurements can be affected by power source variations or bending fiber effects. Instead, the phase modulation has good accuracy and immunity to power intensity variation; unfortunately, ambiguity detection (for instance, thermal sensitivity) and complicated demodu- lation process are involved. It is important to notice that the thermal effects, the experiments were carryout at room temperature. The interferometer was made by splicing a standard single-mode fiber optic and a microfiber (50 $\mu \mathrm{m}$ of diameter). After that, we used the electron beam physical vapor deposition technique to cover the tip fiber optic structure with a thin layer of copper. We compare the interferometer spectral response before and after coating, and it was found that even though for copper-coated interferometers, the sensitivity decreased, the measurement range for solute concentration in aqueous solutions could be increased considerably through minimizing the evanescent field losses.

\section{Fabrication process}

The experimental setup used for the fabrication procedure and its concentration characterization is illustrated in Fig. 1. We use a three-port optical fiber circulator to connect all the elements used to characterize this fiber optic sensor. The incoming signal from the super-luminescent diode (SLD) is routed to Port 2 where the interferometer structure fabrication process happens. The light is reflected at the end of the fiber optic sensor by the reflection at the glass/air interface (Fresnel reflection), and the light is directed to the last port (3) to measures the spectrum using the Optical Spectrum Analyzer (OSA). This scheme is very useful due to allows the monitoring of the fabrication process in real-time.

The microfiber used in this experiment was fabricated using a standard fusion and pulling technique [21]. The parameters were chosen based on the well-established model for 


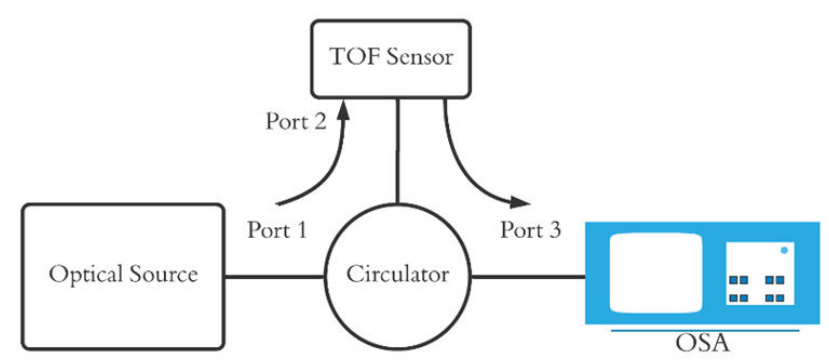

FIGURE 1. The experimental setup used during the fabrication process and the sensing tests. Optical source: super-luminescent diode, OFC: three-port optical fiber circulator, TOF Sensor: Tip Optical Fiber Sensor, OSA: optical spectrum analyzer.

fiber tapers [22]; here, we set a waist of $60 \mu \mathrm{m}$. We use the manufacturing method described in [13] to fabricate the desired interferometer. The microfiber used to generate the interferometer was fabricated using the flame-brushing technique, in which a conventional optical fiber (SMF-28) is fixed on two translational motorized stages. A flame heats the fiber to make it moldable, then a sweep back and forth while the fiber is pulled using the software-controlled motors in the machine. As a result, we obtained a biconical tapered transition and a uniform taper waist. The machine parameters were selected to have a waist of $60 \mu \mathrm{m}$. Using the manual mode of a commercial splicer, we could splice the microfiber and the conventional single-mode fiber. After that, we moved both spliced fibers around $500 \mu \mathrm{m}$ from the electrodes, and we applied about 10 electric discharges to cleave the microfiber. Following this procedure, we obtain an all-fiberoptic interferometer that was operated in reflection mode (see Fig. 2 gray structure). The stretched fiber had approximately $400 \mu \mathrm{m}$ of length.

The tip fiber-optic interferometer (TFOI) before the coating treatment exhibits the interference pattern spectrum observed in Fig. 3 (black curve). The reflected spectrum shows a modulated response. Here the external FSR is related to the first collapsed region generated by the splice between the conventional fiber and the microfiber, and the internal FSR is related to the microfiber length. The free spectral range (FSR) is given by $\lambda^{2} / 2 n d$, where $\lambda$ is the wavelength, $\mathrm{n}$ is the refractive index of the cavity, and $d$ is the length of the cavity; then, the external FSR is about $20 \mathrm{~nm}$, and the internal FSR is about $1.5 \mathrm{~nm}$. For the analyzed wavelength range, the maximal visibility was around $4 \mathrm{~dB}$.

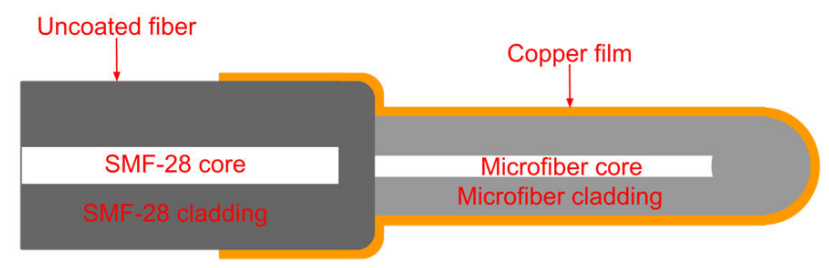

FIGURE 2. Transversal section of the interferometer structure used for sensing. The copper film (orange lines) coated only the tip of the structure.

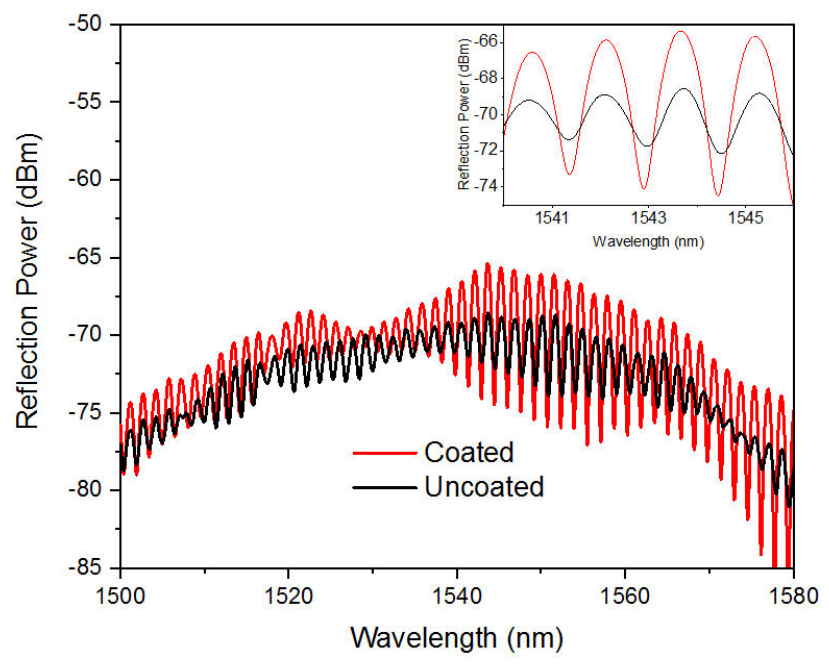

FIGURE 3. Reflection spectrum of uncoated tip fiber optic sensor (black curve) and coated tip fiber optic sensor (red curve). Inset: particular fringe region selected to analyzes the spectral coating effects from $1540 \mathrm{~nm}$ to $1546 \mathrm{~nm}$.

A thin copper film has been symmetrically deposited on the fiber optic structure by the electron beam physical vapor deposition technique using the INTERCOVAMEX H2. The fiber tip was fixed in a sampler holder located approximately $50 \mathrm{~cm}$ over the boat holder where the copper sheet was fixed. To melt the copper, the chamber must be evacuated to a high vacuum, and the working current was set at $130 \mathrm{~A}$ for one minute. This technique was applied two times to coat both sides of the device. Based on similar procedures described at [23], we estimated that the film thickness is about $10-15 \mathrm{~nm}$.

The final interferometer structure is shown in Fig. 2; the orange lines represent the coated film. Here, it is important to point out that the all-fiber-optic interferometer was removed from the fabrication setup (Fig. 1) to carry out this procedure. After that, we reconnected the coated interferometer into the arrangement and observed the spectral response. The newest interference pattern (see Fig. 3, red curve) exhibits a maximal fringe contrast of $12 \mathrm{~dB}, 8 \mathrm{~dB}$ better than the previous one. The simplified formula to calculate the reflectance between two media with refractive index $n_{1}$ and $n_{2}$, is: $R=\left|\left(n_{2}-n_{1}\right) /\left(n_{2}-n_{1}\right)\right|^{2}$. We can approximate for common glass $\left(n_{2} \approx 1.5\right)$ surrounded by air $\left(n_{1}=1\right)$, the power reflectance at normal incidence can be seen to be about 4\% (Fresnel Reflection). The refractive index of copper for $1550 \mathrm{~nm}$ at room temperature is 0.71576 [24,25]. Using the previous equation, we obtain that the reflectance, in this case, is about $13 \%$. Because of that, the copper film often increases the reflectivity and minimizes the evanescent field losses; therefore, the interference pattern spectrum was improved.

Besides by comparing the spectrum of the uncoated and the coated tip interferometer, we can infer that the coating process does not affect the internal FSR. It may occur because the FSR depends on the refractive index of the cavity, 


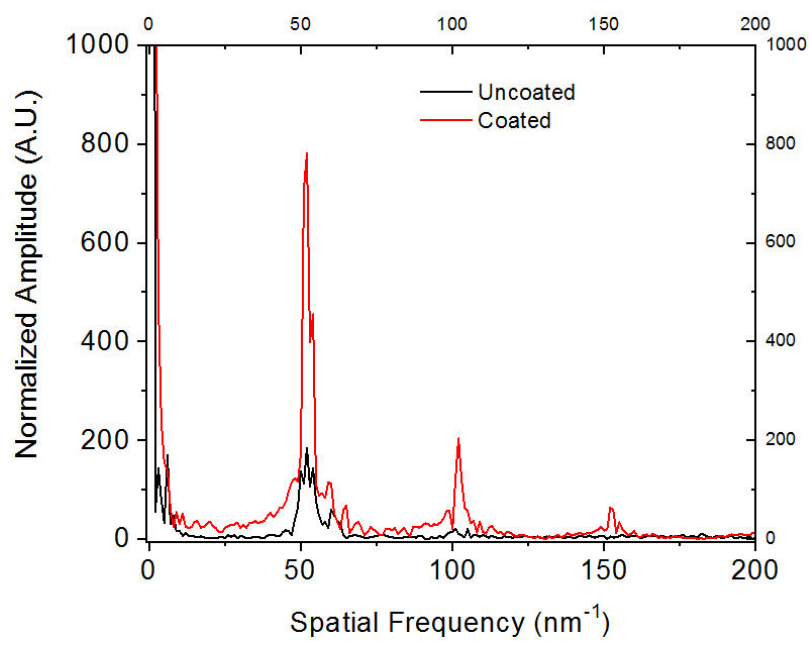

FIGURE 4. Spatial frequency analysis for uncoated tip fiber optic sensor (black curve) and coated tip fiber optic sensor (red curve).

then, the metal coating process does not affect this parameter; however, the external FSR is clearly affected by the metal coating.

As we can appreciate in Fig. 4, the dominant interference spatial frequency is centered at $51 \mathrm{~nm}^{-1}$ for uncoated and coated interferometers, but it is more intense for the coated interferometer.

\section{Principle of operation}

The operation principle of the TFOI was widely described in [13]. Nevertheless, it is necessary to mention that the interference pattern is caused by the interaction between several optical fiber modes generated inside the structure. In Fig. 2, we can appreciate that the TFOI structure is formed by two collapsed regions between the SMF28-collapse regionmicrofiber where refractive index changes may occur. As a result, we can express the reflection interference spectrum obtained by equation [26]:

$$
\begin{aligned}
E_{R} & =\left(\sqrt{R_{1}}+\sum_{k=1}^{n}\left[\exp \left\{-2 j\left(\sum_{i=0}^{k} \phi_{i}\right)\right\}\right.\right. \\
& \left.\left.\times \sqrt{R_{k+1}} \prod_{i=1}^{k}\left(1-R_{i}\right)\right]\right) E_{0},
\end{aligned}
$$

where the reflection can be calculated using the refractive index of the materials presented in the interferometric structure by $R=\left|\left(n_{x}-n_{y}\right) /\left(n_{x}+n_{y}\right)\right|^{2}, R_{1}$ is the reflection at the collapse region between the SMF-28, and the first collapse region and $R_{k+1}$ are the other reflections generated at the collapse region and microfiber interface. The phase between two optical modes generates can be obtained using the expression $\phi=\left(4 \pi n_{x, y} L\right) / \lambda\left(n_{x, y}\right)$ is the effective refractive index of the section involved). The length of the optical path is represented with the letter $L$, and the wavelength is represented with $\lambda$.

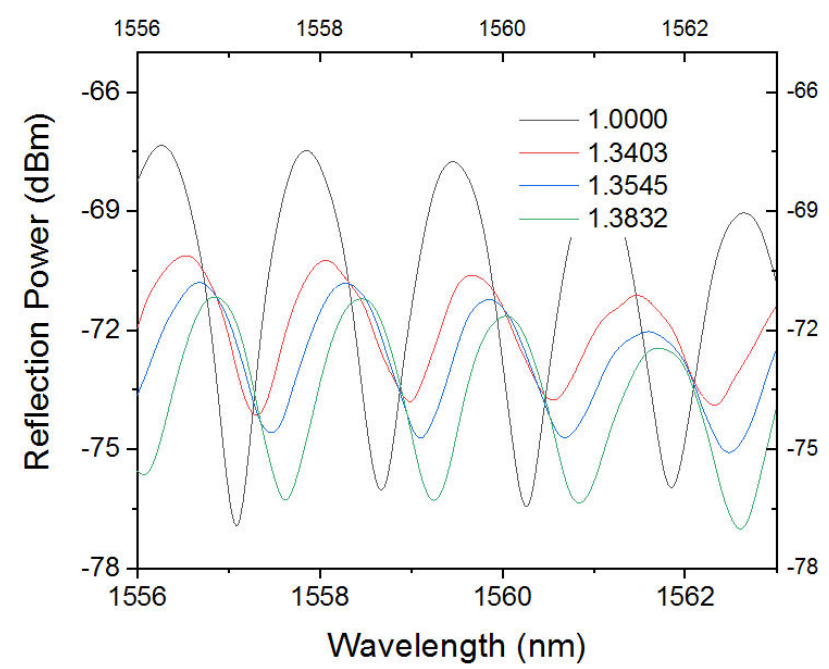

FIGURE 5. Refractive index spectral response of the coated TFOI from 1.0000 to 1.3832 RIU.

\section{Experimental results}

The glycerol solutions were prepared during these experiments using distilled water and pure glycerol. Their refractive index values were ranged from 1.3330 to 1.3903 , the mixtures were estimated by using the refractometer Refracto 30GS METTLER TOLEDO. According to the information presented by [20], it is possible to know the relationship between refractive index and glycerol $\%$ weight concentration.

All the experiments described in this section were realized at controlled room temperature because the reflected spectrum could be affected by changes better than $2^{\circ} \mathrm{C}$ in ambient temperature [19]. Two sets of tests were carried out varying the glycerol concentration in the aqueous solution around the tip sensor: the first was developed after finish the fabrication process, the second round was performed after coated with a thin copper film.

When the glycerol concentration was increased in the aqueous solution around the tip fiber optic sensor, the spectral response shows a red-shifting in both cases (see Fig. 6). Using the initial interferometer, a big intensity modulation can be appreciated because of the evanescent wave. This modulation is affected when the solute concentration in the surrounding aqueous solution increases. When the concentration increased, the spectral response became too flat. A sensitivity up to $27 \mathrm{pm} /$ (Glycerol \% by weight) and a good linear response is showed in this experiment (see Fig. 6 black line). The adjusting linear equation is $\Delta \lambda=$ $-0.00911+0.02683 C$, when $\Delta \lambda$ represents the wavelength shifting, and $C$ represents the solute concentration (Glycerol $\%$ by weight).

On the other hand, when we made the tests using the tip sensor after the coating treatment with copper, we could observe that the pattern visibility was not affected considerably by the surrounded liquid (see Fig. 5), which will allow us to increase the range of measurement until 45 Glycerol \% by weight or even bigger. Wavelength shifting was plotted 


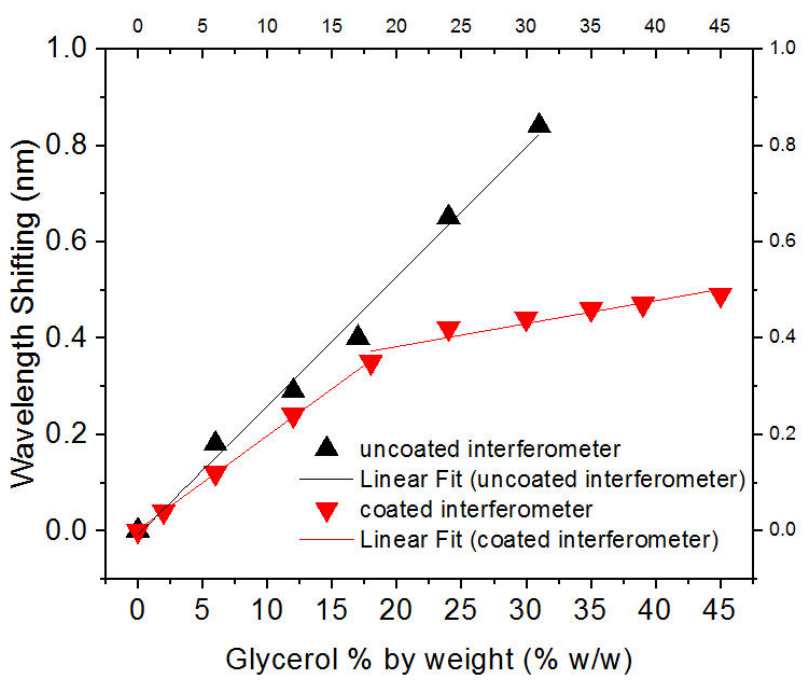

FIGURE 6. Sensitivities analysis of uncoated tip fiber optic sensor (black curve) and coated tip fiber optic sensor (red curve).

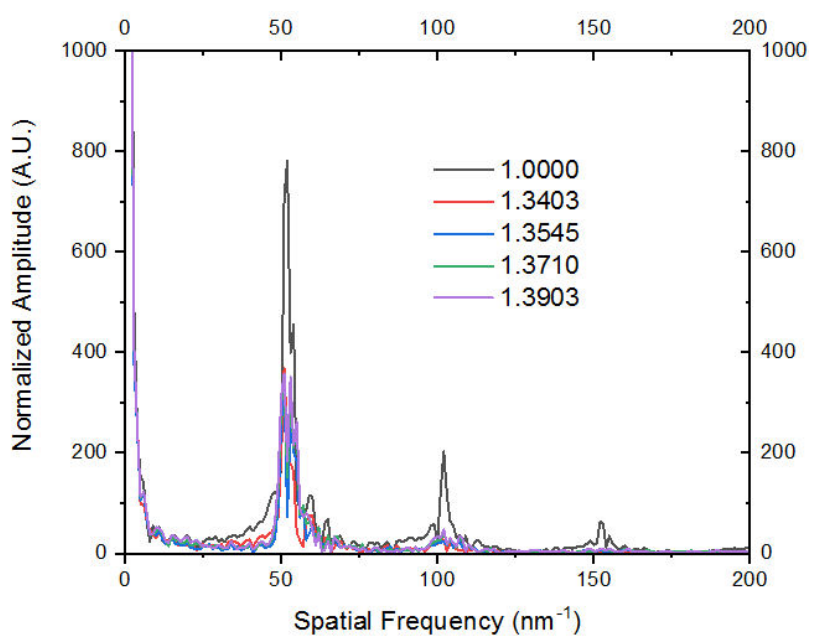

FIGURE 7. Spatial frequency analysis for and coated tip fiber optic sensor when the refractive index was varied from 1.3403 to 1.3903 .

against concentration (see Fig. 6 red line) and found to fall along a curve which could be divided into two sections: a straight line from 0 to 18 Glycerol $\%$ by weight and a straight line from 18 to 45 Glycerol \% by weight, these lines are:

$$
\begin{aligned}
\text { from } 0 \text { to } 18 \% \Delta \lambda=0.00161+0.01953 C, \\
\text { from } 18 \text { to } 45 \% \quad \Delta \lambda=0.28668+0.00476 C .
\end{aligned}
$$

We obtained a sensitivity of $19 \mathrm{pm} /$ (Glycerol \% by weight) from 0 to $18 \%$ and $4.7 \mathrm{pm} /$ (Glycerol \% by weight) from 18 to $45 \%$. The sensitivities obtained in this stage of the work were lower than the previously recorded. By increasing the device reflectivity, minimizing the losses by the evanescent field, the contrast of the interference pattern increases, but the amount of light that interacts with the medium decreases, making the device less sensitive to changes in the concentration of the surrounding medium.
It is important to mention that the surface plasmon resonance (SPR) phenomenon has been widely studied. In some previously reported researches, it has been shown that plasmonic effects can occur in copper-coated structures from UV to NIR region, but the maximum wavelength to observe this effect is 1000 microns [27]. As a result, this effect is neglected in our experiments due to the wavelength operation is higher than the SPR theory.

\section{Discussion}

In this report, we used the electron beam physical vapor deposition technique to cover an all-fiber interferometer with a thin layer of copper. The improved tip fiber-optic interferometer with copper film exhibits maximal visibility of $12 \mathrm{~dB}$; here, a significant increment is achieved by the metal coating. To propose a liquid concentration sensor device, we use different concentration glycerol mixtures and analyzes the sensor spectral responses, first without copper film and after having covered it. The sensitivities for phase modulation detection obtained after the tip was covered were lower than the previously recorded. However, using the copper film, we could increase the range of liquid concentration measurement because the pattern visibility was not affected by the surrounded liquid.

The technique described at [15] allows us to detect the refractive index changes (even at small concentration variation), avoiding the non-linear response obtained using the wavelength analysis previously described; by using the dominant interference spatial frequencies centered at $51 \mathrm{~nm}^{-1}$. As can be appreciated in Fig. 7, the dominant interference spatial frequency centered at $102 \mathrm{~nm}^{-1}$ and $152 \mathrm{~nm}^{-1}$ disappear when the refractive index increases. However, the dominant interference spatial frequency centered at $51 \mathrm{~nm}^{-1}$ did not shift when the refractive index was varied in a controlled way.

When we extracted the phase information from the data and plotted it at an arbitrary wavelength [15] (Fig. 8), we

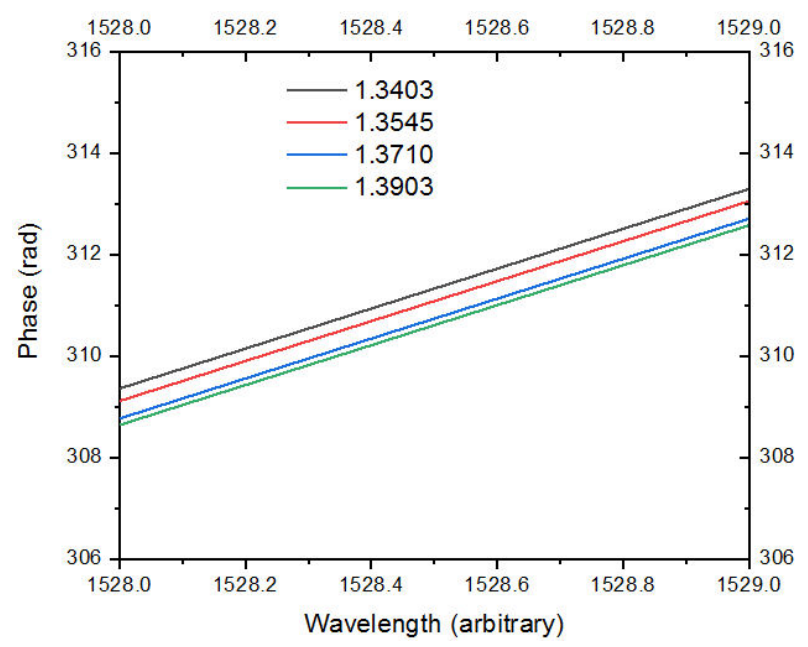

FIGURE 8. Phase changes of the filtered TFOI Fourier component when the refractive index was varied from 1.3403 to 1.3903 . 


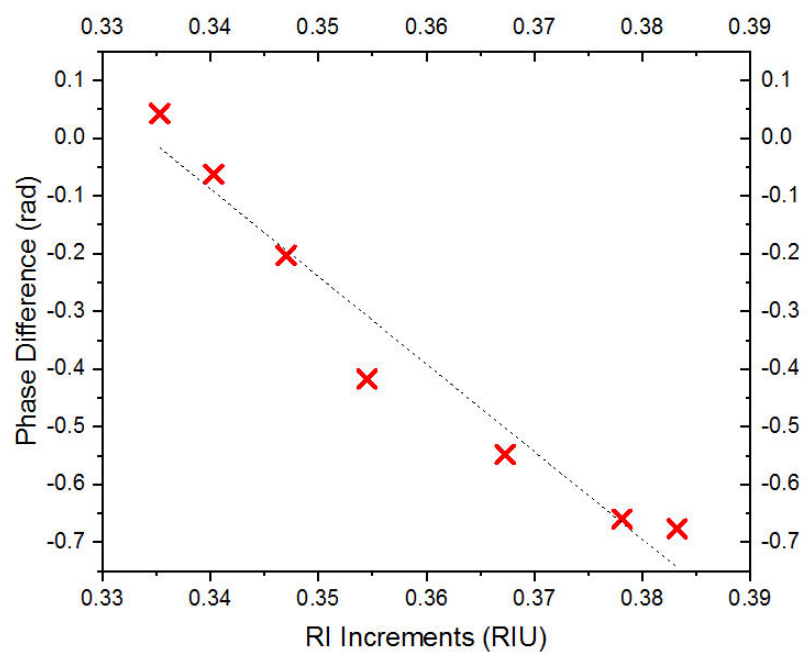

FIGURE 9. Phase difference analysis of the coated TFOI for Refractive Index.

could analyze the phase difference generated when the refractive index was increased. In Fig. 9 can be appreciated that the phase change sensitivity obtained by this technique is about $-15 \mathrm{rad} / \mathrm{RIU}$.

Solute concentration detection in aqueous mixtures is very useful in a lot of industrial processes. This study aims to increase the range of measurement for liquid concentration by minimizing the losses of the evanescent field. Currently, we are directing our effort toward the optimization of the fiber sensor to measure all the range of solute concentration. The proposed sensor can be adapted in several applications in which the aggressive environments would be present, and it is easy to handle by its dimensions.

\section{Conclusions}

In summary, a reflective optical fiber sensor based on a thin copper film was proposed and demonstrated for refractive index variations. In this report, we used the electron beam physical vapor deposition technique to cover an all-fiber interferometer with a thin layer of copper. The improved tip fiber-optic interferometer with copper film exhibits maximal visibility of $12 \mathrm{~dB}$. To propose a liquid concentration sensor device, we use different water-glycerol mixtures, and the spectral responses with copper and without copper were analyzed. The copper film increases the refractive index range measurement. Moreover, by using the phase information from the FFT analysis, we obtained a sensitivity of $-15 \mathrm{rad} / \mathrm{RIU}$. The proposed sensor can be adapted in several applications in which the aggressive environments would be present, and it is easy to handle by its dimensions.

\section{Acknowledgments}

The authors would like to thank UG for the project grant CIIC 238/2020, Secretaría de Educación Pública, through PRODEP, with a post-doctorate support, and SICES under project AI-20-37. This work was supported by the Consejo Nacional de Ciencia y Tecnología (CONACYT) under Grant $577494 / 307127$.

In memoriam of Prof. E. A. Kuzin.
1. W.P. Chen, D.N. Wang, B. Xu, C.L. Zhao, H.F. Chen, Multimode fiber tip Fabry-Perot cavity for highly sensitive pressure measurement, Sci. Rep. 7 (2017) 1. doi $10.1038 /$ s41598-017-00300-x

2. W. Wang, Z. Mai, Y. Chen, J. Wang, L. Li, Q. Su, X. Li, X. Hong, A label-free fiber optic SPR biosensor for specific detection of C-reactive protein, Sci. Rep. 7 (2017) 1. doi $10.1038 /$ S41598-017-17276-3

3. N. Zhong, Q. Liao, X. Zhu, M. Zhao, Y. Huang, R. Chen, Temperature-independent polymer optical fiber evanescent wave sensor, Sci. Rep. 5 (2015) 1. doi 10 .1038/srep11508

4. T. Okazaki, T. Orii, A. Ueda, A. Ozawa, H. Kuramitz, Fiber Optic Sensor for Real-Time Sensing of Silica Scale Formation in Geothermal Water, Sci. Rep. 7 (2017) 1. doi $10.1038 /$ s41598-017-03530-1

5. Z. Lin, R. Lv, Y. Zhao, H. Zheng, High-sensitivity salinity measurement sensor based on no-core fiber, Sensors Actuators A Phys. (2020) 111947. doi $10.1016 /$ j.sna.2020. 111947

6. F. Zhao, J. Wang, Y. Xiao, K. Zhang, R. Chen, S. Liu, Curvature monitoring of power grid wires based on anti-resonant reflect- ing guidance in hollow core fibers, Optik (Stuttg). 213 (2020) 164785. doi $10.1016 / j . i j l e o .2020 .164785$

7. E. Vorathin, Z.M. Hafizi, N. Ismail, M. Loman, Review of high sensitivity fibre-optic pressure sensors for low pressure sensing, Opt. Laser Technol. 121 (2020). doi $10.1016 / j$. optlastec.2019.105841

8. J. Hribar, D. Donlagic, Fiber-Optic Boiling Point Sensor for Characterization of Liquids, IEEE Sens. J. 20 (2020) 7731. doi 10.1109 /JSEN.2020.2982420

9. M.H. Jali, H.R.A. Rahim, M.A.M. Johari, S.S. Hamid, H.H.M. Yusof, S. Thokchom, P. Wang, S.W. Harun, Optical characterization of different waist diameter on microfiber loop resonator humidity sensor, Sensors Actuators A Phys. 285 (2019) 200. doi $10.1016 / j . s n a .2018 .11 .025$

10. N. De Acha, C. Elosúa, J.M. Corres, F.J. Arregui, Fluorescent sensors for the detection of heavy metal ions in aqueous media, Sensors (Switzerland). 19 (2019). doi $10.3390 /$ S19030599

11. J. Zhou, Y. Wang, C. Liao, B. Sun, J. He, G. Yin, S. Liu, Z. Li, G. Wang, X. Zhong, J. Zhao, Intensity modulated refractive index sensor based on optical fiber Michelson interferometer, 
Sensors Actuators, B Chem. 208 (2015) 315. doi $10.1016 /$ j.snb.2014.11.014.

12. P. Chen, X. Shu, H. Cao, K. Sugden, Ultra-sensitive refractive index sensor based on an extremely simple femtosecond-laserinduced structure, Opt. Lett. 42 (2017) 1157. doi $10.1364 /$ OL.42.001157.

13. Y. Lopez-Dieguez, J.M. Estudillo-Ayala, D. Jauregui-Vazquez, L.A. Herrera-Piad, J.M. Sierra-Hernandez, J.C. HernandezGarcia, M. Bienchetti, J.R. Reyes-Ayona, R. Rojas-Laguna, Tip fiber-optic intermodal interferometer for refractive index sensing, IEEE Photonics Technol. Lett. 30 (2018) 15. doi 10. $1109 / \mathrm{LPT} .2017 .2771409$.

14. S.S.J.R.C. Yee; A fiber optic chemical sensor based on surface plasmon resonance, Sensors Actuators B Chem. 12 (1993) 213. doi $10.1016 / 0925-4005$ (93) 80021-3.

15. D. Jauregui-Vazquez, J.W. Haus, A.B.H. Negari, J.M. SierraHernandez, K. Hansen, Bitapered fiber sensor: Signal analysis, Sensors Actuators B Chem. 218 (2015) 105. doi $10.1016 / j$. snb.2015.04.109.

16. M. V. Hernández-Arriaga, M.A. Bello-Jiménez, A. RodríguezCobos, M. V. Andrés, Experimental Investigation of Fused Biconical Fiber Couplers for Measuring Refractive Index Changes in Aqueous Solutions, IEEE Sens. J. 16 (2016) 132. doi $10.1109 /$ JSEN.2015.2475320.

17. Y. Zhao, L. Cai, X.G. Li, F.C. Meng, Liquid concentration measurement based on SMS fiber sensor with temperature compensation using an FBG, Sensors Actuators, B Chem. 196 (2014) 518. doi:10.1016/j.snb.2014.01.075.

18. Y. Zhao, Z.Q. Deng, Q. Wang, Fiber optic SPR sensor for liquid concentration measurement, Sensors Actuators, B Chem. 192 (2014) 229. doi $10.1016 / j$.snb.2013.10.108 .
19. H. Su, X.G. Huang, Fresnel-reflection-based fiber sensor for on-line measurement of solute concentration in solutions, Sensors Actuators B Chem. 126 (2007) 579. doi $10.1016 / j$. snb.2007.04.008.

20. J. Segur, Physical properties of glycerol and its solutions, Aciscience.Org. (1953) 1.

21. B.S. Kawasaki, K.O. Hill, R.G. Lamont, Biconical-taper singlemode fiber coupler, Opt. Lett. 6 (1981) 327. doi $10.1364 /$ OL.6.000327.

22. T.A. Birks, Y.W. Li, The Shape of Fiber Tapers, J. Light. Technol. 10 (1992) 432. doi $10.1109 / 50.134196$.

23. C. Cobianu, M.F. Stan, I. Bancuta, N. Fidel, INVESTIGATION OF Ni-Cu THIN FILMS MAGNETIC SENSORS DEPOSITED ON SiO 2 SUBSTRATES BY SPUTTERING, $J$. Sci. Arts. 4 (2019) 1055.

24. P.B. Johnson, R.W.Christy, Optical Constants of the Noble Metals, Phys. Rev. B. 6 (1972) 4370. doi $10.1016 /$ j.susc. 2018.02 .016

25. $\mathrm{Cu}$ Refractive Index Database, (n.d.). https: //refractiveindex. info/?shelf=main\&book= Cu\&page=Johnson

26. Y. Lopez-Dieguez, J.M.M. Estudillo-Ayala, D. JaureguiVazquez, J.M.M. Sierra-Hernandez, L.A.A. Herrera-Piad, J.M.M. Cruz-Duarte, J.C.C. Hernandez-Garcia, R. RojasLaguna, Multi-mode all Fiber Interferometer based on FabryPerot Multi-cavity and its Temperature Response, Opt. Int J. Light Electron Opt. 147 (2017) 232. doi $10.1016 / j$. ijleo.2017.08.091

27. G.H. Chan, J. Zhao, E.M. Hicks, G.C. Schatz, R.P. Van Duyne, Plasmonic Properties of Copper Nanoparticles Fabricated by Nanosphere Lithography, Nano Lett. 7 (2007) 1947. doi 10 . $1021 / \mathrm{n} 1070648 \mathrm{a}$ 